\begin{tabular}{|l|l|}
\hline La simultaneidad en la historia global & Titulo \\
\hline Rabasa, José - Autor/a; & Autor(es) \\
\hline $\begin{array}{l}\text { De Raíz Diversa. Revista Especializada en Estudios Latinoamericanos (Vol. 2 no. 3 } \\
\text { ene-jun 2015) }\end{array}$ & En: \\
\hline México D.F. & Lugar \\
\hline $\begin{array}{l}\text { Programa de Posgrado en Estudios Latinoamericanos, Universidad Nacional } \\
\text { Autónoma de México }\end{array}$ & Editorial/Editor \\
\hline 2015 & Fecha \\
\hline Schmitt, Carl; Tiempo; Espacio; Historia; México; América Latina; & Colección \\
\hline Artículo & Temas \\
\hline "http://biblioteca.clacso.edu.ar/Mexico/ppel-unam/20160614071054/Rabasa.pdf" & Tipo de documento \\
\hline $\begin{array}{l}\text { Reconocimiento-No Comercial-Sin Derivadas CC BY-NC-ND } \\
\text { http://creativecommons.org/licenses/by-nc-nd/2.0/deed.es }\end{array}$ & URL \\
\hline
\end{tabular}

Segui buscando en la Red de Bibliotecas Virtuales de CLACSO http://biblioteca.clacso.edu.ar

Consejo Latinoamericano de Ciencias Sociales (CLACSO)

Conselho Latino-americano de Ciências Sociais (CLACSO)

Latin American Council of Social Sciences (CLACSO)

www.clacso.edu.ar

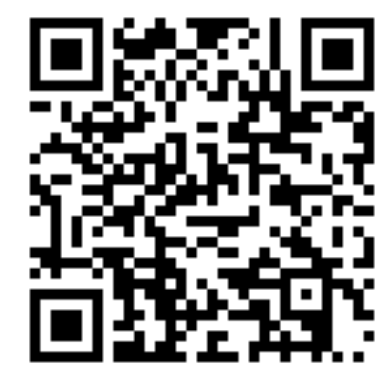




\section{La simultaneidad en la historia global}

JosÉ RABASA *

Resumen. El trabajo cuestiona que el llamado Mapa de Cuauhtinchan núm. 2 , pueda ser analizado, desde el presente y con la carga de valores culturales occidentales, como un mapa en estricto sentido. El propósito de este ensayo es discutir aquellas visiones, como las de Carl Schmitt, que plantean una universalidad de la "apropiación, distribución, y producción" del nomos, apuntando hacia una actitud que apueste por la pluralidad de las formas de representar los distintos espacios-tiempos. En este sentido el autor avanza en la manera de proponer distintas categorías que permitan una mejor comprensión de los documentos pictográficos que se produjeron antes y durante la conquista de Mesoamérica y las demás latitudes que fueron colonizadas por los españoles.

Palabras Clave: Nomos, elsewhere, espacio-tiempo, simultaneidad.

Aвstract. The work questions the call Cuauhtinchan map. No. 2, it can be analyzed from the present and the burden of Western cultural values as a map strictly. The purpose of this essay is to discuss those visions, like those of Carl Schmitt, posed a universality of "appropriation, distribution, and production" nomos, pointing an attitude that is committed to the plurality of ways of representing the different areas-Times. In this sense the author advances in the way of proposing different categories that allow a better understanding of the pictorial documents that occurred before and during the conquest of Mesoamerica and other latitudes that were colonized by the Spaniards.

KEYWORD: Nomos, elsewhere, space-time, simultaneous.

Recibido: 13 de octubre de 2014. ACEPTAdo: 11 de diciembre de 2014 .

$\mathrm{E}$

arqueo-astrónomo Anthony F. Aveni concluye su contribución a la reproducción del Mapa de Cuauhtinchan núm. 2 (fig. 1) y recopilación de ensayos a cargo de Davíd Carrasco y Scott Sessions, en Cueva, ciudad y nido de águila, con las siguientes preguntas:

¿Era el MC2 un relato histórico enmarcado en un texto didáctico concebido para el chamán novicio? ¿Era un panorama portátil concebido para acompañar al peregrino? ¿Se trataba de un documento legal que formaba parte de un proceso judicial? ¿O era algo que está fuera del alcance de nuestra imaginación colectiva? (156).

1 Profesor emérito de la Universidad de California, Berkeley <rabasajose@gmail.com> 
Las preguntas de Aveni nos llevan a reflexionar sobre los límites de los universales con los que nos aproximamos al MC2. Entre los universales figurarían las reflexiones en varios de los ensayos interpretativos en Cueva, ciudad y nido de águila sobre el chamanismo a partir de Mircea Eliade (Carrasco y Sessions, " 'El Lugar del Medio' laberinto y circunvalación," García Goyco, "El árbol cósmico"), el uso de la narratología para identificar prácticas historiográficas (Boone, "La casa del águila"), la noción de que el MC2 es una historia de corte cartográfico o, para el caso, que es un mapa (Seiferle-Valencia, "Representaciones de organización territorial"). Podríamos a su vez pensar el MC2 a partir de las estructuras universales jurídicas identificadas por Carl Schmitt en su libro The Nomos of the Earth, tema a desarrollar en este ensayo. El propósito de este ensayo es precisamente el de interrumpir una tal reflexión a través de una lectura del MC2. Más adelante discutiré las propuestas de Schmitt sobre la supuesta universalidad de los procesos de apropiación, distribución, y producción que Schmitt elabora en su Part V. Appendix: "Three Concluding Corollaries" en The Nomos of the Earth in the International Law of Jus Publicum Europeaum. Para este efecto la yuxtaposición del MC2 y las propuestas de Schmitt dan lugar a un cuestionamiento de los a priori (la universalidad de la apropiación, distribución, y producción) que informan su historia del nomos supuestamente englobante de la totalidad del mundo y la historia: "In every stage of social life, in every economic order, in every period of legal history until now, things have been appropriated, distributed, and produced" (Schmitt, 2006: 327).

La dificultad del cuestionamiento de la universalidad, por ende, de la aplicabilidad de los conceptos de Schmitt reside en que debemos proceder sin establecer prácticas mesoamericanas con datos positivos. La última pregunta de Aveni es, a mi parecer, la más sugerente en tanto que nos llama la atención sobre la necesidad de pensar el espacio y el tiempo de la producción del MC2 en términos de un elsewhere, de un "algo que está fuera del alcance de nuestra imaginación colectiva”, para adoptar los términos de Aveni. ${ }^{1}$ Su carácter de elsewhere nos fuerza a pensar en la co-existencia de varias concepciones espacio-temporales de la historia,

1 Por elsewhere no debemos entender un otro que implicaría irremediablemente un mismo, sino un espacio-tiempo con intencionalidad propia que debemos dejar que nos habite (véase Rabasa, Tell Me the Story...). Tampoco debemos entender un opuesto a la razón, es decir un irracional frente al que debemos desarrollar un método para su explicación e interpretación. Dejo estas observaciones sin desarrollar. 
en lo que siguiendo a François Hartog denominaríamos régimes d'historicité. Si bien siempre ya participamos de espacios comparativos y son inevitables las categorías occidentales con las que nos manejamos, y que en última instancia determinan nuestras traducciones o adopciones de vocablos nahuas, podemos dejar que el MC2 y otros textos mesoamericanos nos guíen en nuestra reflexión. Si los debates en el contexto de la historia de Europa ya dan lugar a un cuestionamiento de los a priori de Schmitt, el MC2 adquiere su pertinencia en esta discusión en tanto que el punto de partida en la historia del nomos de Schmitt son los escritos de Francisco de Vitoria sobre la legalidad de la conquista y apropiación de las Américas. El MC2 nos ofrece la posibilidad de pensar las tesis de Vitoria desde la experiencia de un pueblo nahua.

La distinción entre mapa y territorio es un lugar común que nos recuerda que no debemos confundir los modelos de la realidad con la realidad misma; sin embargo, en el caso del MC2 el modelo define un territorio invisible para cualquier lector occidental. El MC2 delinea los elementos naturales de las narrativas históricas de una primera migración de los antepasados remotos chichimeca de los Cuauhtinchantlaca desde Chicomoztoc y la posterior expedición tolteca desde Cholula para solicitar la ayuda de los chichimecas (Boone, 2011: 28-29). Las siete cuevas de Chicomoztoc, el lugar de origen de todos los pueblos nahuas, se encuentra en la parte superior izquierda del MC2, desde la cual se puede trazar la migración al centro de México. Itzpapalotl (la mariposa de obsidiana) guía a los chichimecas en su surgimiento de Chicomoztoc. En el centro del MC2 encontramos Cholula, ciudad a la que adoptaron los toltecas después de la caída de Tula alrededor de 1150. Dos líderes tolteca viajan a Chicomoztoc a pedir ayuda a los chichimecas para combatir a los antiguos residentes de Cholula, los olmeca-xicalanca. La Historia tolteca-chichimeca, otro texto de Cuauhtinchan que combina pictografía y alfabeto, registra el tipo de cantos, narraciones, gritos, monólogos, y descripciones que se darían en una lectura de las expediciones-migraciones pintadas en el MC2. Todos estos lugares pintados en el MC2 pueden ser visitados y recordados en el caminar de los itinerarios. Nos encontramos con un desajuste adicional en tanto que el mundo de estas migraciones ha sido destruido por la invasión europea. Se da una paradoja ya que un mundo supuestamente inexistente materialmente retiene su presencia y contemporaneidad en un mapa que excede al territorio. Esta paradoja de lo contemporáneo en la que la 
captación de un territorio cuya realidad depende de la repetición de los itinerarios y las narrativas nos recuerda que los muertos son más reales que los vivos.

La inclusión en el MC2 de dos iglesias y de un templo prehispánico que ha sido destruido (fig. 2) se presta para una reflexión sobre la permanencia de mundos no apropiables. Hablo de inclusión para evitar el lenguaje de la re-presentación que supondría que el objetivo del MC2 fue la creación de una imagen objetiva del mundo. Esto no quiere decir que la iglesia a la izquierda del clip del mapa no tenga elementos que podamos identificar con la estructura del ex-convento franciscano de San Juan Bautista aún hoy observable en Cuauhtinchan. Tampoco importa si la estructura urbana junto a la iglesia a la derecha corresponde o no al espacio circundante. Las iglesias, o para el caso la distribución de las casas, manifiestan la capacidad de generar nuevos signos afines al vocabulario pictórico mesoamericano para denotar objetos europeos. Estas iglesias hacen patente el hibridismo del MC2. Más allá de estas iglesias podemos hablar de formas pictóricas que identificaríamos como europeas, por ejemplo, los usos del sombreado y el alfabeto latino en la palabra escrita junto a los dos sujetos, un joven y un viejo, en el exterior de la iglesia. Hibridismo que no le quita su carácter mesoamericano. Tampoco se trata de ver el MC2 formando parte de un proceso indefinido de mestizaje y globalización. Más adelante precisaré la diferencia entre historia global y globalización histórica.

Varios autores de los ensayos en Cueva, ciudad y nido de águila, así como en otros estudios de códices coloniales, han recalcado que el uso de textos pictóricos tenía tanta, o más autoridad que la escritura alfabética en las cortes españolas. En el caso del MC2, sin embargo, la yuxtaposición de un templo pagano junto a la iglesia católica sugiere que éste era un texto para consumo interno. Para 1544 (según los cálculos de Keiko Yoneda este es el año que corresponde a la fecha interna del glifo 13 tecpatl [Yoneda, 2011: 190]) ya se habían destruido los templos paganos así que la inclusión del referente ahora imaginario de una continuidad del pasado mesoamericano, sin correspondencia en la realidad fuera de los escombros que aún pudieran ser identificados, no sería bien vista ni por las autoridades 
eclesiásticas ni por las seculares. ${ }^{2}$ Carecería de toda pertinencia para la argumentación sobre los derechos territoriales de Cuauhtinchan. Su uso del MC2 dentro de la comunidad para rememorar las tradiciones de Cuauhtinchan hasta entrado el siglo veinte implica la continuidad de los relatos que han acompañado las lecturas del MC2 y la posibilidad de pensar desde un elsewhere no moderno las transformaciones socio-económicas y políticas (los procesos de apropiación, distribución y producción de Schmitt) que ha vivido Cuauhtinchan en la colonia y durante los gobiernos republicanos. La comunidad de Cuauhtinchan hasta la fecha retiene otros documentos paralelos al MC2.

En la yuxtaposición de la iglesia y el templo encuentro una articulación de una simultaneidad espacio-temporal. Más allá de la simultaneidad de lo católico y lo pagano, se daría la noción un tanto paradójica de que el MC2 (a manera de instancia mesoamericana) y sus posibles articulaciones verbales sugieren una existencia fuera de la historia colonial o republicana que determinaría sus significados. Esta paradoja implica la contemporaneidad del significado del MC2 con la pluralidad de momentos en los que se ha leído, cantado, caminado e interpretado. Estas lecturas sitúan al MC2 en un presente continuo. La destrucción de Cuauhtinchan no es algo que ocurrió hace mucho tiempo sino que se da en en un presente vivido en las repeticiones de los relatos. La realidad del MC2 es contemporánea al tiempo de la reflexión. Aun cuando el MC2 fue extraído del archivo Cuauhtinchan (evito palabras más fuertes) las formas de leer, cantar, caminar que se practicaron mientras el MC2 permaneció en la comunidad continúan siendo practicadas con documentos existentes en ese archivo. ${ }^{3}$ El concepto de contemporaneidad es ahistórico si no por naturaleza, por vocación-exige que suspendamos toda noción de tiempo formulada en términos de secuencias de instantes o momentos. La ahistoricidad de la contemporaneidad implica la posible existencia de múltiples "lecturas" simultáneas-por ejemplo, la hermenéutica del historiador académico y el caminar rememorando de la comunidad. Mientras que un lector à la Schmitt (o para el caso que asuma la aplicabilidad de los conceptos y ca-

\footnotetext{
2 Según Ethelia Ruiz Medrano no debemos excluir el hecho de que habiendo sido primero producido para el consumo interno de la comunidad el mapa haya sido posteriormente refuncionalizado para presentarlo "ante las cortes españolas ya pasado el tiempo y cuando los españoles no prestaban particular atención a las escenas del culto idolátrico" (Ruiz, 2011: 109).

3 Véase el panorama histórico que elabora Ruiz Medrano en "Los señores de la tierra".
} 
tegorías hoy día vigentes en las ciencias sociales) encontraría pruebas de sus principios universales en los linderos y otras señales que sugerirían formas de propiedad, la comunidad en su caminar reiteraría la convicción de la existencia de un mundo poblado de seres con agencia propia, y por lo tanto no apropiables. No podemos insistir demasiado sobre las especificidades de los múltiples regímenes políticos e históricos en Mesoamérica. ${ }^{4}$ Volveré sobre esta cuestión. Por ahora precisemos las dificultades que encontramos al hablar de simultaneidad.

Una definición física de la simultaneidad requiere que la coexistencia de eventos se dé en un mismo instante. Siempre queda pendiente la cuestión sobre qué perspectiva define el instante que incluye a los diferentes eventos. Insisto en la noción de instante ya que toda lectura, reflexión o interpretación se da en un instante cuya duración puede ser variable. La duración nos permite hablar de diferentes formas de vivir el instante y por lo tanto de experimentar el MC2. Los académicos que han estudiado el MC2 pueden muy bien compartir la temporalidad de su discusión con miembros de la comunidad de Cuauhtinchan. Esta sería una circunstancia particularmente fructífera para una reflexión sobre la contemporaneidad del MC2. Dicho esto, dudo que los presentes dueños del mapa estén dis-

4 En la Tercera relación y aún más insistentemente en el Memorial breve acerca de la fundación de la ciudad de Culhuaca, el historiador chalca Domingo Chimalpahin marca alternancias entre los regímenes de tlahtohuani y el mando del cuauhtlatollo. Víctor M. Castillo traduce cuauhtlatolli por mandato rústico, literalmente "habla de monte o salvaje" (Chimalpahin, 1991: 48-49). Cuautlatoa significa gritar pero también ejercer un gobierno militar. ¿Sugiere esta denominación un gobierno menos jerárquico al fundado en el lenguaje, es decir, en el régimen político del tlahtohuani? Obsérvese que en la narrativa de Chimalpahin se da una alternancia entre los regímenes. Quizás sea un exceso hablar de formas de propiedad comunal que nunca desaparecieron a pesar de las formaciones de regímenes de propiedad ligados a los pilli, los pillalli (tierras de los señores, los pilli, concepto que en la época colonial definirá los cacicazgos) que según James Lockhart se dan en el polo opuesto al calpolli, tierras pertenecientes a individuos pero bajo la supervisión de la comunidad (156). Uno de los efectos de la colonización fue la disolución de las diferencias entre los pillalli y los calpolli que reduce a todos los indígenas al estatus de macehuales, muy a pesar de los cacicazgos que hasta la fecha corrompen el espíritu comunal de las comunidades indígenas en México. A mi parecer la formación de los pillalli no destruyó el comunitarismo originario sino que este continuó existiendo en los calpolli. Mi objetivo no es negar las jerarquías en los regímenes de propiedad en la antigüedad mesoamericana sino simplemente sugerir que en un momento "rústico" se daba un régimen comunal que nunca dejó de existir en su totalidad y que puede justificar hablar de una continuidad temporal, es decir, de la contemporaneidad con lo que hoy día se conoce por las tierras comunales y los ejidos. 
puestos a regresarlo a la comunidad de Cuauhtinchan y de ahí dar lugar a una lectura en un espacio y tiempo irreducible a la de la producción académica.

En el MC2 la simultaneidad se da en varios planos. Estos incluyen la coexistencia de dos memorias, de la desaparecida existencia de los templos y de la implantación del cristianismo. Los templos fueron desmantelados y sus "sacerdotes" perseguidos, conducidos a la clandestinidad. Los templos no solo nos remiten a un pasado cercano sino a la posibilidad de pensar el presente cristiano a partir de cronologías indígenas o, por adoptar la terminología bajtiniana, tan bien implementada por Federico Navarrete, de los cronotopos mesoamericanos. La simultaneidad se daría por lo menos a partir de dos referentes temporales: por un lado, a partir de las cuentas del xiuhpoalli y el tonalpoalli, por el otro, de la cuenta del anno domini cristiano. Los significados serían radicalmente diferentes ya que se asumiría que estos calendarios, a pesar de la tendencia a establecer meras correspondencias, tendrían significados propios. Referirse al anno domini no se limita a dar una mera fecha, ni tampoco lo es referirse al ciclo mesoamericano de cincuenta y dos años. La referencia al anno domini vendría desde fuera, desde un observador, ya que en el MC2 no encontramos ninguna fecha europea.

En el MC2 encontramos las dos temporalidades y la posibilidad de pensar lo global en tanto que la evangelización tenía como marco de referencia la expansión del cristianismo a nivel mundial. Las iglesias indicarían la entrada de Cuauhtinchan a la historia, es decir, a la historia universal judeocristiana. Pero a la vez la yuxtaposición con el templo pagano permite pensar a Cuauhtinchan fuera de esta historia, y aún sin historia. Es un lugar común insistir en la historicidad de los pueblos amerindios para subrayar las transformaciones que estas sociedades han vivido y continúan experimentando. Su carácter histórico evitaría constituir esencias inmutables. Sin embargo, mi objetivo no es el de establecer una esencia, sino el de precisar que en el MC2 se da una afirmación de lo destruido que se niega a confirmar el éxito de la conquista y la evangelización. El mundo que ha dejado de existir permanece en el imaginario del MC2. Este es un mundo imaginario que puede ser reconstituido y recordado a partir de los restos en el paisaje. Esto implica un sin historia en tanto que el régime d'historicité del MC2 debe ser pensado aparte de los supuestos universales de la historia. El gesto caritativo que identifica los textos indígenas como 
modalidades de historia no deja de ser problemático. La atribución de la historia a los pueblos amerindios implica la proyección de formas de pensar y de constituir el pasado que no son transferibles. Se ignora que la leyenda, el mito, los sueños y la imaginación en general son formas de rememoración del pasado, presente y futuro.

II

Desde la perspectiva del MC2 los argumentos de Francisco de Vitoria recapitulados por Carl Schmitt parecerían un tanto gratuitos en tanto que la comunidad en su destrucción resiste cualquier reflexión sobre la justicia o injusticia de la apropiación española. Los argumentos de Vitoria se dan a partir de una abstracción jurídica que para los sujetos de Cuauhtinchan no puede ser más que un abuso. Esta yuxtaposición de Vitoria cum Schmitt y el MC2 hace patente la simultaneidad de temporalidades y marcos de referencia divergentes. La simultaneidad en la historia global implica el reconocimiento de diferentes tradiciones memorísticas coexistiendo independientemente en múltiples localizaciones del planeta. ${ }^{5}$ Recordemos que De Indis y De Jure belli Hispanorum in barbaros, ambos escritos por Francisco de Vitoria, habiéndose escrito apenas una década antes, son contemporáneos al MC2. En su yuxtaposición manifiestan simultaneidades que hacen patente la necesidad de pensar los procesos en la historia global desde perspectivas irreducibles a una historia única. Por un lado, Vitoria establece los criterios a partir de los cuales se condenan o justifican las guerras de conquista desde un escenario jurídico internacional, por el otro, el MC2 define la memoria del espacio temporal de Cuauhtinchan desde una comunidad que ha sido destruida. El MC2 hace patente que las memorias no han sido borradas, y aún menos las formas de recuerdo e inscripción del espacio. Pero más allá de estas formas culturales, los

5 Este fue el proyecto del 3er tomo del Oxford History of Historical Writing, pero como ya lo precisamos los editores en la introducción al tomo 3, la producción de esta historia se ve viciada por las fechas 1400-1800 que enmarcan los ensayos dedicados al Japón, China, Medio Oriente y las Américas. Este reclamo de fechar a partir del sistema anno domini o, para el caso, de la era común se puede repensar a partir de la simultaneidad en el MC2. Especifico el 3er tomo porque la posibilidad de pensar la globalización se establece en la transición de los años que irían del 1400 al 1492, fecha que define la periodización predilecta de las teorías de los sistemas-mundo. Teorías de sistemas-mundo, que a diferencia de la historia global que acentúa la coexistencia de régimes d'historicité, presuponen una globalización totalizante cuando no, un proceso de mestizaje universal. 
documentos de Cuauhtinchan (no el MC2, sino otros "mapas" de linderos) se podrían utilizar en sus litigios dentro del horizonte legal del régimen imperial español.

El espacio legal español se presta al menos a dos opciones interpretativas: 1) la excepcionalidad del imperio español: el hecho de que la llamada pax hispana diera lugar a una participación indígena en las cortes; 2) la participación en el sistema jurídico español promovió la asimilación de las sociedades indígenas a la racionalidad occidental (ver Ruiz Medrano y Kellogg). No es mi objetivo discutir estas opciones, tampoco el de situarme dentro de una de ellas, sino recordar que las teorías jurídicas sobre la conquista tienen sus correspondientes articulaciones (si bien no teóricas) indígenas. Esta simultaneidad expone la fatuidad del derecho internacional que desde sus inicios se ha limitado a expresiones formales sin vigencia en el plano real. Es decir, sin una aplicación efectiva aparte del beneficio que derivan los países, sectores e individuos en el poder: desde el "obedezco, pero no cumplo" de la colonia, al "me paso por las narices" las convenciones de Ginebra en las recientes guerras de Estados Unidos. Observemos que el formalismo de Schmitt da lugar a una historiografía sin fisuras. Indica el fin del eurocentrismo en las denuncias del colonialismo generalizadas después de la segunda guerra. Parecería que Schmitt lamenta la disolución del orden espacial del Jus Publicum Europaeum que solo cubría la paz entre los países europeos sin ninguna preocupación por la destrucción de los territorios sujetos por las potencias europeas. Las preocupaciones de Vitoria sobre los "justos títulos" de la conquista han sido desplazadas por un derecho internacional exclusivamente pertinente a Occidente. Interacción que queda fuera de los principios que eventualmente, según la narrativa de Schmitt, van a definir la novedad del Jus Publicum Europaeum en términos de una aplicabilidad restringida a los países europeos.

A partir de un giro historicista, Schmitt sitúa el derecho anterior al secularismo del Jus Publicum Europaeum en la prehistoria. ${ }^{6}$ Tanto el derecho español del siglo xvi como el que queramos atribuirle a Mesoamérica, argumentaría Schmittt, está viciado por entes metafísicos, mágicos o teológicos. Pero ésta no es la problemática de Schmitt que nos concierne sino

6 Sería más preciso hablar de países cristianos. Véase la denuncia del cristianismo solapado en el secularismo de Edward Said en Anidjar. 
la de establecer la validez del a priori de la universalidad de la apropiación del mundo. Regresaré más adelante a esta cuestión.

III

Nos debemos preguntar si en la larga historia de los performances del MC2 (y otros documentos de Cuauhtinchan) las iglesias cristianas no serían lugares para una meditación sobre la existencia de la comunidad dentro y fuera de la historia de la salvación. La posibilidad de pensar las iglesias desde un fuera de la historia implicaría un elsewhere a partir del cual se podría reconceptualizar la globalización inherente a la evangelización de todos los pueblos del planeta. La exterioridad a la historia universal del cristianismo también implicaría que la comunidad y su memoria no fueron necesariamente absorbidas por la aparente lógica totalizadora de la modernidad. Este planteamiento requiere de una reflexión sobre la posibilidad de pensar lo moderno y lo no moderno como contemporáneos.

Lo moderno se define a partir de regímenes de verdad: la racionalidad occidental que produce otredades al reducirlas a la superstición, el fetichismo, la idolatría. Estas categorías son aplicadas no solo a partir de los dogmas religiosos sino también de las ciencias naturales y la economía del capital. Por muy incipiente que sea la ciencia en el siglo dieciséis ésta no deja de operar en la colonización de las Américas. La ciencia tiene como vocación separarse de estadios anteriores que son definidos como pre-científicos. Como lo he indicado anteriormente en la historia del derecho de Schmitt se establecen las formas jurídicas con bases teológicas o míticas en la prehistoria del Jus Publicum Europaeum. Las modalidades teológicas o míticas no dejan de existir sino que son desestimadas. En este sentido siempre se da una coexistencia de lo moderno y lo no moderno.

En el caso del MC2 la coexistencia de lo moderno y lo no moderno se manifiesta en la continuidad de la antigüedad mesoamericana en un espacio imaginario que ha dejado de existir al ser reducido a ruinas por el furor destructivo de la colonización. Este furor destructivo es un resultado de la violencia evangelizadora que destruye todo rastro de paganismo, pero también de la apropiación económica y política que niega la vigencia de los entes imaginarios recordados por el mapa. Las narrativas, cantos y gritos que los recordarían en un caminar del espacio hace patente la contemporaneidad de lo moderno (la constante destrucción) y lo no moderno 
(la permanencia de formas de vida). Lo moderno implica la necesidad de negociar cuando no litigar en las cortes a partir del régimen jurídico español. Este régimen jurídico da cabida a "usos y costumbres" bajo el principio de jus gentium pero que en última instancia implica apelar a los derechos a partir de los códigos españoles que constituyen los criterios de su universalidad, es decir, su compatibilidad con las leyes de todas las naciones. La participación en las cortes conllevaría la producción de documentos, mapas y argumentos articulados para un escenario español, algo que no se da en el MC2. ${ }^{7}$ Podemos especular que el mismo sujeto, por individualizar la producción del MC2, podría ser responsable de la factura de ambos tipos de documentos. Dada su condición de sujetos colonizados la participación de Cuauhtinchan en la modernidad no es una opción; sin embargo, el MC2 hace patente la existencia de la comunidad en un espacio ajeno a los regímenes de verdad y apropiación europeos.

El proyecto de pensar la simultaneidad en la historia global se puede resumir a un esfuerzo por pensar lo global fuera de la globalización totalizante y su historia en clave singular. Los procesos globalizadores implican la producción de temporalidades históricas distintas para su destrucción. El énfasis recae en la absorción de todas las formas de vida en una condición espacio-temporal sin exterioridad. En este proceso las heterogeneidades históricas tendrían sus días contados. Debemos sin embargo hacer una distinción entre las otredades producidas por la ciencia y la religión, y las formas de vida que no pueden ser reducidas a las definiciones de los regímenes de verdad modernos.

Tomemos por ejemplo el caso del historiador de la India, Dipesh Chakrabarty, que si bien identifica la coexistencia del tiempo de la historia y los tiempos de los dioses, su argumento lleva a la conclusión de que los tiempos de los dioses carecen de toda viabilidad frente a las instituciones modernas, ya sean éstas el Fondo Monetario Internacional o el Estado. ${ }^{8}$ En la formulación del binario se da una lógica que establece la otredad como un dato empírico y no un producto discursivo. Dentro de estos parámetros no existe ninguna duda de que la incompatibilidad es algo evidente. Sin embargo, no debemos descartar el hecho de que la contemporaneidad de

Para estudios y reproducciones de mapas producidos en Cuauhtinchan que se presentan en las cortes, véase Reyes García (1978); Yoneda (2011) reproduce los mapas de Cuauhtinchan 1, 3, y 4 en Los mapas de Cuauhtinchan.

8 Para una crítica de Chakrabarty más elaborada, véase Rabasa, "Epilogue: Before History". 
los tiempos permita un transitar entre múltiples mundos sin incurrir en contradicción. Tampoco debemos asumir que todo discurso de memoria, por no privilegiar la disciplina histórica en la remembranza del pasado, está pensado para construir argumentos frente al estado o las instituciones financieras que controlan la distribución del capital.

La simultaneidad de diferentes tipos de mapas en las comunidades mesoamericanas, por ejemplo el MC2, para el consumo interno y otros mapas diseñados para presentar argumentos en las cortes, implica un saber muy claro sobre la necesidad de las diferentes formas de presentar los linderos y el pasado de la comunidad. Las articulaciones del derecho internacional de Vitoria que proponen un orden del mundo tienen su contraparte en los documentos nahuas producidos fuera y dentro de las interacciones con el estado colonial. Si bien la litigación en las cortes coloniales conlleva una posible normalización de los nahuas en los documentos que producen, documentos como el MC2 hacen patente una permanencia fuera de las estructuras del derecho español. El ordenamiento del mundo en su totalidad que según Schmitt se da por primera vez en el siglo dieciséis acarrea la destrucción de mundos en los procesos de apropiación:

Vitoria's doctrine of just war made posible the appropriation of foreign, non-free land. The many conquests, surrenders, occupations, annexations, cessions, and successions in world history either fit into an existing spatial order of international law, or exceed its framework and have a tendency, if they are not just passing acts of brute force, to constitute a new spatial order of international law. (Schmitt, 2006: 82)

Schmitt parece ofrecernos una versión del binario de Chakrabarty. Por un lado, presupone el tiempo de la historia en el proceso de apropiación, por el otro, los foreign, non-free lands estarían bajo los tiempos de los dioses destinados a desaparecer. La fuerza de la globalización lleva a la constitución de un nuevo orden de derecho internacional. A pesar del sentido único de la historia de Schmitt, sabemos que los sujetos de los non-free lands aprendieron las nuevas reglas del juego sin abandonar sus concepciones del mundo.

Para Chakrabarty la heterogeneidad de los tiempos de los dioses necesitaría en última instancia del pensamiento del historiador moderno para una negociación con el Estado. El historiador produce saberes para el uso 
de los trabajadores sociales. ${ }^{9}$ La mediación viene de fuera. No existe la posibilidad de que un sujeto pueda vivir en la simultaneidad de ambas temporalidades.

En todo caso se trata de encontrar un acercamiento para establecer una familiarización con la temporalidad heterogénea. En el presente la dependencia de las comunidades subalternas en el trabajo del historiador se hace patente en la incapacidad de coexistir en lo moderno y lo no moderno. Es todo una cuestión de tiempo antes de que lo heterogéneo sea absorbido en una singularidad histórica. El sujeto de la heterogeneidad histórica carece de los medios para pensar el Estado. El binomio que yuxtapone el tiempo de la historia a los tiempos de los dioses parecería aproximarse al sentido de una coexistencia o contemporaneidad, pero al menos en el caso de Chakrabarty, el gesto va más hacia la negación de la viabilidad de los tiempos de los dioses en la aparentemente ineludible modernidad globalizadora. Si podemos hacer una distinción entre globalización hegemónica y alterna, esta diferenciación por muy prometedora que sea para la eventual creación de un mundo más justo, no deja de serlo al costo de la especificidad de las historias plurales existiendo en una simultaneidad diferencial. Si bien los tiempos de los dioses carecen de viabilidad en el contexto de las instituciones modernas, y según Chakrabarty están condenados a desaparecer, el $\mathrm{MC} 2$ da constancia de mundos poblados por seres y entidades que supuestamente han dejado de "existir" en Occidente, al menos desde los límites de la epistemología kantiana.

9 Podemos ligar esta formulación de los saberes de los historiadores y los trabajadores sociales con el fenómeno que Isabelle Stengers ha denominado "the curse of tolerance", la maldición de la tolerancia: "Nothing is easier for modern man than tolerance. How could it be otherwise? How could we not be tolerant? I am not referring here to 'others, to those in whom we encourage tolerance. I'm speaking of 'we, and this 'we' does not refer to a concrete group to which one may or may not belong, but to all recipients of the message of modernity." (303). En este punto parecería que Stengers coincide con Chakrabarty en tanto que la recepción del mensaje de la modernidad implica la transformación de los sujetos. No existe la posibilidad de transitar entre lo moderno y no moderno. En los términos de Stenger, "They are not supposed to generate knowledge themselves, but merely produce modifications in relationships, enabling negotiation whenever there is a threat of confrontation or repression" (Stengers, 2010: 305). La diferencia entre Stengers y Chakrabarty reside en que ella se plantea la necesidad de establecer una salida a la tolerancia. 
IV

En el MC2 encontramos un mundo poblado por seres que la racionalidad europea ha expulsado del horizonte de la experiencia. No podemos asumir que entre los nahuas se concibieran los procesos de población y constitución de linderos en los términos de "apropiación, distribución, y producción" que Schmitt atribuye a todas las sociedades. La apropiación del mundo requiere de una epistemología en la que el mundo es inerte, en el que no existan seres a los que hay que solicitar permiso, es decir, hacer los rituales y sacrificios necesarios para convivir con ellos. La coexistencia de un templo sagrado junto a los dos templos cristianos en el MC2 sugiere que este mapa no fue producido para hacer reclamos territoriales en las cortes españolas. La inclusión del templo pagano para ese entonces destruido tiene un paralelismo con los lugares sagrados, los sacrificios, y las entidades naturales y sobrenaturales con las que interactuaban los cuauhtinchantlaca en la vida cotidiana y en sus recorridos del territorio definido por el MC2. Lejos de ser un mero mapa de linderos para reclamar derechos territoriales en las cortes coloniales, el MC2 establece una memoria regida por una epistemología en la que todo acto de apropiación del mundo carece de sentido.

Resisto llamarla "epistemología indígena" por el simple hecho de que esta terminología desembocaría en una dicotomía que respondería, que sería una reacción a la imposición de una definición de la epistemología europea. Prefiero utilizar el concepto de epistemología desarrollado por Gregory Bateson en su libro Mind and Nature, un magnífico ejercicio en la disolución de las dicotomías que han viciado al pensamiento occidental: "It seemed to me that in 'Schoolboy' I was laying down very elementary ideas about epistemology, that is, about how we can know anything. In the pronoun we, I of course included the starfish and the redwood forest, the segmenting egg, and the Senate of the United States" (Bateson, 2010: 4). Bateson evita toda definición de la epistemología en abstracto al privilegiar las entidades mismas en sus adquisiciones de saberes. Nos encontramos con un nosotros que incluye estrellas de mar, huevos segmentándose, árboles sequoia, en fin, todas las formas de vida posibles. Bateson excluye la materia física, pero no veo por qué no podemos incluirla a la manera en que las deidades mesoamericanas, los teules, literalmente las "cosas" 
extraordinariamente buenas o malas, se manifiestan en los ojos de agua, las montañas, las piedras.

La definición de Bateson abre la posibilidad de concebir múltiples sujetos interactuando en espacios comunes. La apropiación como práctica universal desata una violencia que se desenvuelve en tres momentos: 1) se niega la existencia de seres que observan nuestra voluntad de dominio; 2) estas formas de aprehensión son sistemáticas, es decir, asumen un habitus que ignora la arbitrariedad de la apropiación del mundo; 3) la apropiación ignora la simultaneidad de las múltiples temporalidades vigentes en cualquier momento global que no ha sido reducido a una globalización hegemónica o alterna. La esperanza de la globalización alterna es, en última instancia, la realización de un devenir hegemónico. La apropiación al fin de cuentas solo se retiene por la fuerza de las armas o del dinero. Esto puede ser muy cierto-sin embargo, mi objetivo ha sido el de describir mundos en los que se dan simultaneidades que manifiestan elsewheres que nos permiten entender lo global sin incurrir en la globalización. Estos mundos, más o menos vulnerables, pueden ser sujetos a procesos interpretativos y explicativos que desembocan en la apropiación intelectual de corte académico. Aquí me he limitado a sugerir una forma de aproximarnos al MC2 u otros artefactos mesoamericanos desde una relación que no aspira, es más, que vigila ese habitus tan inculcado en las disciplinas que asumen que no podemos pensar de otra forma que a través de la apropiación hermenéutica.

Más allá de esta circunspección frente a las ciencias humanas y sociales, este artículo se ha propuesto cuestionar los a priori de Schmitt sobre la universalidad de las categorías de la "apropiación, distribución, y producción" que tenderían a naturalizar los proyectos imperialistas europeos. El MC2 me ha permitido pensar las condiciones coloniales de los pueblos de las Américas fuera de los esquemas globalizadores que asumen la existencia de una historia singular. En la simultaneidad de la historia global se hacen manifiestas las miradas de todos esos entes que han existido y continúan existiendo fuera de los regímenes políticos e históricos de occidente. Quedan por verse las consecuencias del violentar el mundo de todo proceso de apropiación. 


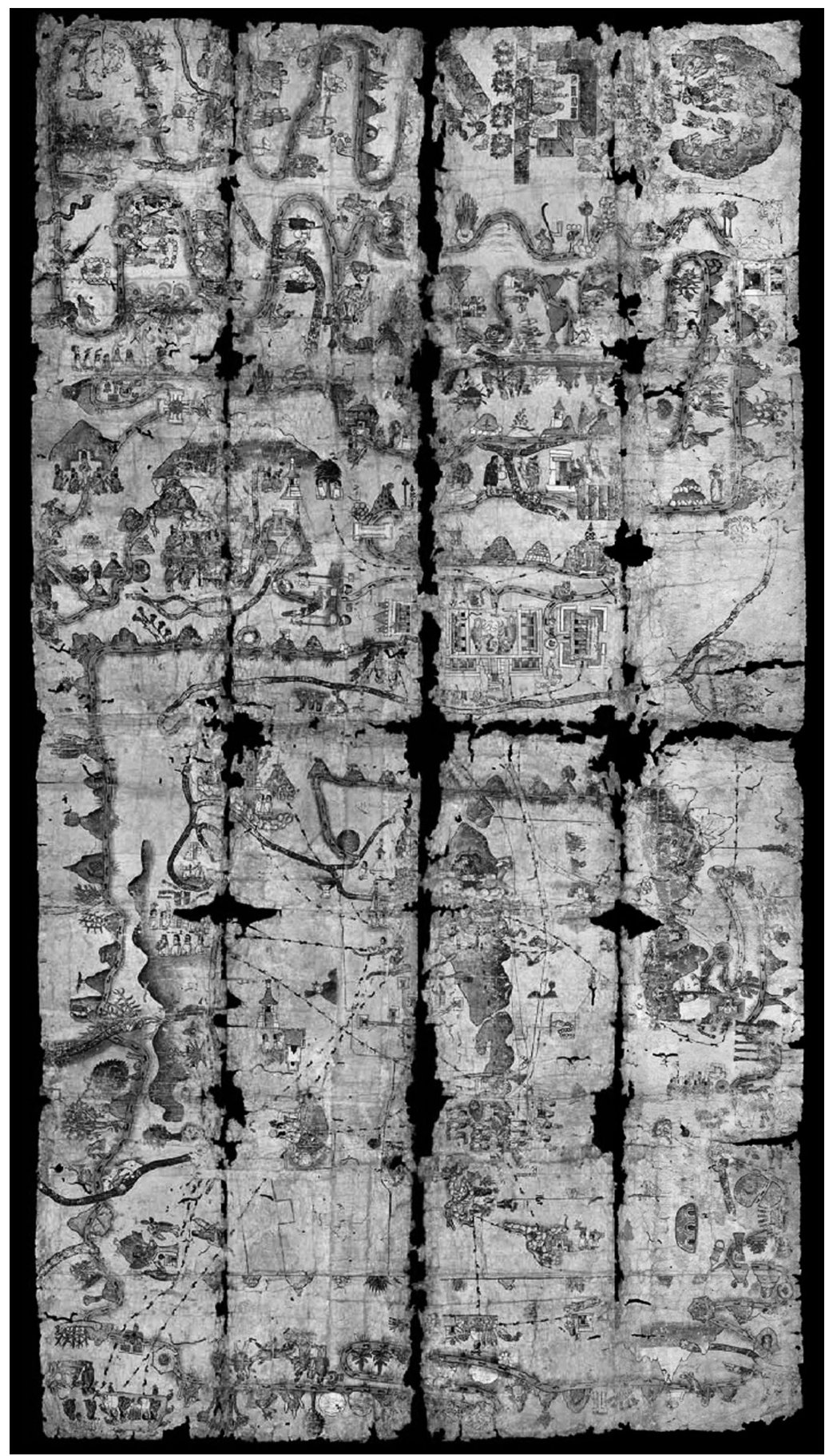

Figura 1. Mapa de Cuauhtinchan núm. 2 


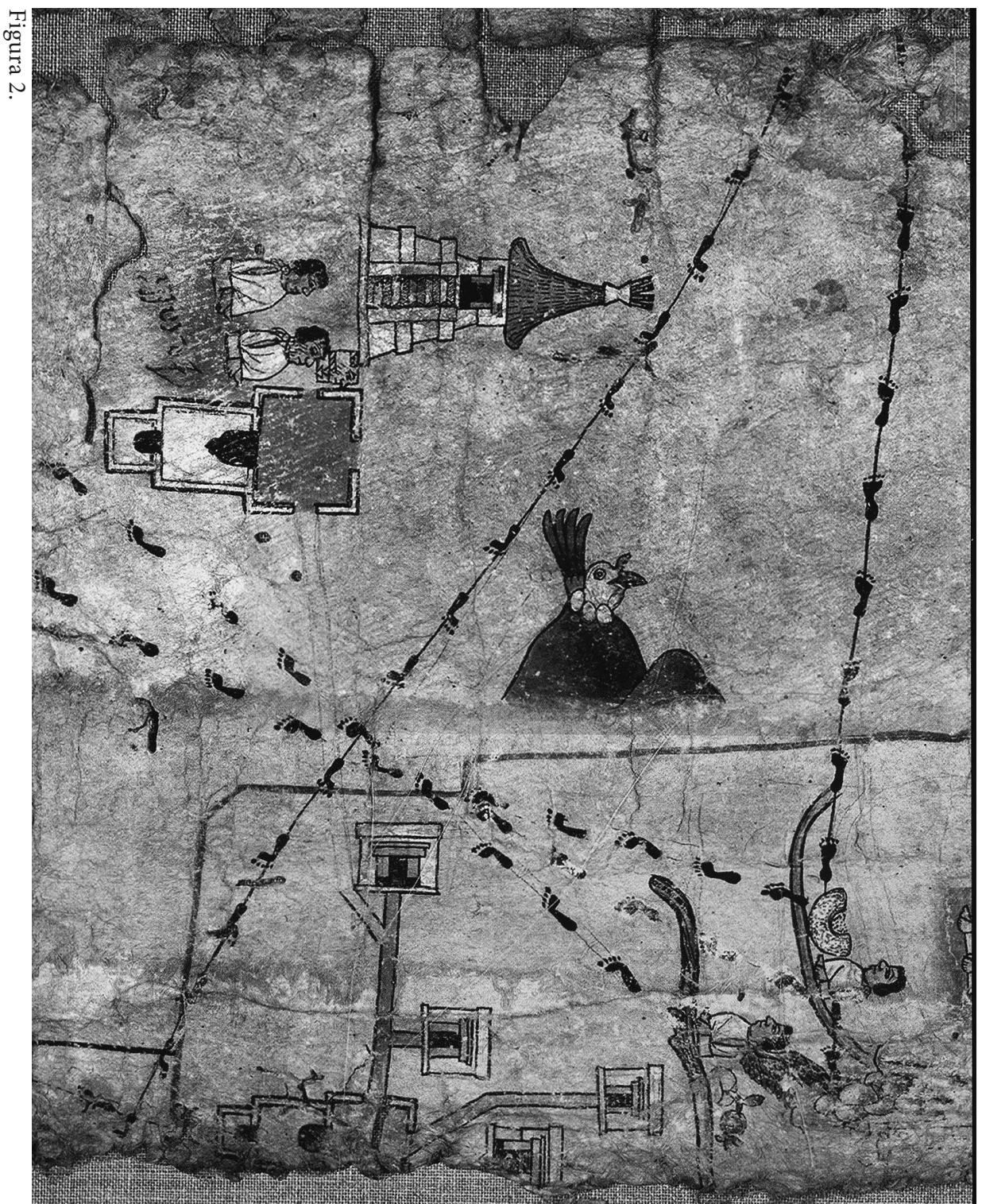




\section{BIBLIOGRAFÍA}

ANIDJAR, G. (2006); “Secularism” en Critical Inquiry, Núm. 33, pp. 52-77.

AVENI, A. F. (2011); “Calendario, cronología y cosmología”, en D. Carrasco y S. Sessions (Eds.) Cueva, ciudad y nido de águila. Una travesía interpretativa por el Mapa de Cuauhtinchan núm. 2. Albuquerque: University of New Mexico. Carasco y Sessions, pp. 147-158.

BATESON, GREGORY (2002), Mind and Nature: A Necessary Unity. Cresskill, NJ: Hampton Press.

BOONE, E. H. (2011); "La casa del águila”, en D. Carrasco y S. Sessions (Eds.) Cueva, ciudad y nido de águila. Una travesía interpretativa por el Mapa de Cuauhtinchan núm. 2. Albuquerque: University of New Mexico, pp. 27-47.

CARRASCO, D. y SESSIONS, S. ; “'Lugar del Medio,' laberinto y circunvalación. El papel peripatético de Cholula”, en D. Carrasco y S. Sessions (Eds.) Cueva, ciudad y nido de águila. Una travesía interpretativa por el Mapa de Cuauhtinchan núm. 2. Albuquerque: University of New Mexico, pp. 427-53.

CHAKRABARTY, D. (2011); "The Time of History and the Times of the Gods", en L. Lows y D. Lloyd (ed.) The Politics of Culture in the Shadow of Capital. Durham: Duke UP, pp. 35-60.

CHIMALPAHIN QUAUHTLEHUANITZIN, D. F.(2003), Las ocho relaciones y el memorial de Colhuacan. Ed. y trad. Rafael Tena. 2 vols. México: CONACULTA.

(1991); Memorial breve acerca de la fundación de la Ciudad de Culhuacan, Ed. y trad. Víctor M. Castillo. México: Universidad Nacional Autónoma de México.

GARCÍA GOYCO, O. (2011); “El Mapa de Cuauhtincan núm. 2 y el árbol cósmico en Mesoamérica. El Caribe y la cuenca del Amazonas y Orinoco”, en D. Carrasco y S. Sessions (Eds.) Cueva, ciudad y nido de águila. Una travesía interpretativa por el Mapa de Cuauhtinchan núm. 2. Albuquerque: University of New Mexico, pp. 357-87.

HARTOG, F. (2012); Régimes d'historicité. Présentisme et expérience du temps. Paris: Seuil.

Historia tolteca-chichimeca (1989); P. Kirchhoff, L. O. Güemes y L. Reyes García (ed. y trad.). México: CIESAS/FCE/GEP.

LOCKHART, J. (1992); The Nahuas After the Conquest: A Social and Cultural History of the Indians of Central Mexico, Sixteenth Through Eighteenth Centuries. Stanford: Stanford UP.

NAVARRETE LINARES, F. (2011); Los orígenes de los indígenas de México. Los altépetl y sus historias. México: Universidad Nacional Autónoma de México. 
RABASA, J. (2000); “Epilogue: Before History”, en Writing Violence on the Northern Frontier: The Historiography of Sixteenth-Century New Mexico and Florida and the Legacy of Conquest. Durham: Duke UP, pp. 275-84.

(2011); Tell me the Story of How I Conquered You: Elsewheres and Ethnosuicide in the Colonial Mesoamerican World. Austin: University of Texas.

(2012); SANTO, M.; TORTAROLO, M. Y WOOLF, D. The Oxford History of Historical Writing. Vol. 3: 1400-1800. Oxford: Oxford UP.

REYES GARCÍA, L. (1978); Documentos sobre tierras y señoríos en Cuauhtinchan. México: INAH-CIS.

RUIZ MEDRANO, E. (2011); “Los señores de la tierra. El contexto histórico del Mapa de Cuauhtinchan núm. 2", en D. Carrasco y S. Sessions (Eds.) Cueva, ciudad y nido de águila. Una travesía interpretativa por el Mapa de Cuauhtinchan núm. 2. Albuquerque: University of New Mexico, pp. 91-119.

RUIZ MEDRANO, E. y KELLOGG, S. (Eds.) (2010); Negotiation within Domination: New Spain's Indian Pueblos Confront the Spanish State. Boulder: University of Colorado.

SCHMITT, C. (2006); The Nomos of the Earth in the International Law of Jus Publicum Europeaum. Trad. G. L. Ulmen. New York: Telos Press Publishing.

SEIFERLE-VALENCIA, A. C. (2011); "Representaciones de la organización territorial en el Mapa de Cuauhtinchan núm. 2", en D. Carrasco y S. Sessions (Eds.) Cueva, ciudad y nido de águila. Una travesía interpretativa por el Mapa de Cuauhtinchan núm. 2. Albuquerque: University of New Mexico, pp. 81-90.

STENGERS, I. (2010); Cosmopolitics, 2 vols, Trad. Robert Bononno. Minnesota: University of Minnesota.

VITORIA, F. (1963); Las relecciones De Indis y De iure belli, Ed. Javier Malagón Barceló. Washington: Unión Panamericana.

YONEDA, K. (2011); "Glifos y mensajes en el Mapa de Cuauhtinchan núm. 2. Chicomoztoc, Itzpapalotl y 13 Tecpatl”, en D. Carrasco y S. Sessions (Eds.) Cueva, ciudad y nido de águila. Una travesía interpretativa por el Mapa de Cuauhtinchan núm. 2. Albuquerque: University of New Mexico, pp. 161203.

(1981); Los mapas de Cuauhtinchan y la historia cartográfica prehispánica. México: Archivo General de la Nación. 